\title{
RIQUEZA DE ESPÉCIES DE SUB-BOSQUE DE UM FRAGMENTO DE FLORESTA OMBRÓFILA MISTA EM TIJUCAS DO SUL, PR ${ }^{1}$
}

\section{RICHENESS OF SPECIES OF SUB-CANOPY IN FRAGMENT IN MIXED OMBROPHILOUS FOREST OF TIJUCAS DO SUL, PR}

\author{
Dieter Liebsch $^{2}$ Luiz Antonio Acra ${ }^{3}$
}

\section{RESUMO}

Este estudo foi realizado em um fragmento com 6 ha sob predomínio de Floresta Ombrófila Mista no município de Tijucas do Sul, PR. Teve como objetivo a confecção de um catálogo de espécies de subbosque e avaliação dos impactos de perturbações antrópicas sobre a composição deste estrato florestal. A coleta de material botânico se estendeu de outubro de 2001 a setembro de 2002, com coletas mensais. Em duas parcelas de $100 \times 100 \mathrm{~m}$, foram identificadas 89 espécies e 54 gêneros distribuídos em 32 famílias botânicas, sendo 84 espécies para a área A (capoeira lato sensu) e 27 espécies para a área B (floresta). Na capoeira as famílias mais ricas foram Solanaceae, com 16 espécies, Asteraceae (15) e Melastomataceae (14). Na floresta, as famílias mais ricas foram Melastomataceae com seis espécies, Solanaceae (cinco) e Myrtaceae (cinco). O predomínio de espécies das famílias Solanaceae e Asteraceae na capoeira demonstra o caráter pioneiro que essas famílias desempenham na recomposição de florestas antropizadas. A presença da Família Solanaceae na floresta é marcante na composição das trilhas. Tais dados demonstram que para esse fragmento a regeneração está ocorrendo e para que esse processo obtenha sucesso, a área deveria ter o seu acesso restringido.

Palavras-chave: florística; pioneiras.

\section{ABSTRACT}

This study was done in a 6 ha fragment of Mixed Ombrophilous Forest in the county of Tijucas do Sul, - Paraná. Its aim was the elaboration of a sub-canopy species catalog and evaluation of the perturbation of anthropic impacts on the composition of this forest stratum. Collection of botanical material was conducted monthly from October 2001 until September 2002. Two sample units of 100 x $100 \mathrm{~m}$. Were usid and 89 species, 54 genera distributed into 32 botanical families were identified, belonging to 84 species in area A (shrub) and 27 species in area B (forest). In the shrub area the most representative families were Solanaceae, with 16 species, Asteraceae (15) and Melastomataceae (14). In the forest area the most frequent families were Melastomataceae with 6 species, Solanaceae (5) and Myrtaceae (5). The predominance of species from Solanaceae and Asteraceae in the shrub area demonstrates the pioneer character of these families, playing an important role in the re-establishment of this forest. Species of the Solanaceae family in the forest were most abundant along the footpaths inside the forest. The data showed that for this fragment, regeneration is successfully taking place and the continuation of this process includes restricted access to the area.

Key words: floristic composition; pioneer species.

\section{INTRODUÇÃO}

A Floresta Ombrófila Mista, mais conhecida como Floresta de Araucária ou Pinheiral por apresentar uma presença massiva do pinheiro-do-paraná, pinheiro-brasileiro, pinheiro ou simplesmente araucária (Araucaria angustifolia (Bert.) O. Kuntze) (Gerhardt et al., 2001), já revestiu grande parte do território paranaense, representando uma das mais ricas formações florestais do Estado, bem como do planalto sulbrasileiro (Koehler et al., 1998; Klein, 1960, 1984; Reitz e Klein, 1966). Segundo Lindmann (1974) a araucária é a maior de todas as árvores florestais do sul do Brasil e por sua grandiosidade e expressiva dominância, tornou-se símbolo do emblema do estado do Paraná (Maack, 1968).

1. Trabalho financiado pelo PELD/CNPq.

2. Biólogo, Pontifícia Universidade Católica do Paraná, Rua Luiza Lelia Gulin Geronasso, 475 CEP: 82650-260 Boa Vista, Curitiba, Paraná. E-mail: dliebsch@bol.com.br

3. Professor, Pontifícia Universidade Católica do Paraná, Rua Imaculada Conceição, 1155 CEP: 80215-901 Prado Velho, Curitiba, Paraná. E-mail laacra@ rla01.pucpr.br

Recebido para publicação em 27/7/2001 e aceito em 17/12/2002. 
A densidade das araucárias geralmente é baixa (Roseira, 1990; Negrelle e Silva, 1992; Jarenkow e Baptista, 1987; Longhi, 1980), permitindo o desenvolvimento de um sub-bosque de latifoliadas tropicais (Backes et al., 2000). A composição florística do sub-bosque dessas florestas varia de uma região para outra (Reitz e Klein, 1966; Backes, 1988, 1999; Fernandes e Backes, 1998). Espécies de sub-bosque são aquelas que completam todo seu ciclo de vida no interior da floresta (Oliveira et al., 2001). Para Finol apud Pizatto (1999), essas espécies apresentam características próprias, mas nunca chegam a passar do piso inferior, sendo pouco desenvolvidas em altura e muito tolerantes à sombra.

Atualmente, segundo Britez et al. (2000), restam menos de 1\% de Floresta com Araucária em boas condições de conservação no estado do Paraná, e a situação atual desse importante bioma é altamente crítica. Relictos de floresta nativa intactos são esparsos e raríssimos, sobretudo em Curitiba e em seus arredores (Koehler et al., 1998). O conhecimento de quais espécies compõem uma determinada floresta, o quanto cada espécie se acha presente e como ela se distribui pela comunidade, é indispensável para que um plano de aproveitamento, com propostas para conservação e manejo desses habitats, seja bem sucedido (Oliveira e Rotta, 1982).

Esse trabalho teve como objetivos a elaboração de uma lista de espécies de sub-bosque de um remanescente de Floresta Ombrófila Mista, comparando a riqueza e a composição florística de duas áreas de diferentes estágios de perturbações antrópicas e de regeneração, propiciando, dessa forma, um maior conhecimento e embasamento para estudos futuros.

\section{MATERIAL E MÉTODOS}

\section{Área de estudo}

O estudo foi desenvolvido no Sistema Ecológico VIVAT FLORESTA, uma área com 5084 ha, $\left(25^{\circ} 45^{\prime} \mathrm{e} 26^{\circ} 00^{\prime} \mathrm{S} / 49^{\circ} 20^{\prime}\right.$ e $49^{\circ} 05^{\prime} \mathrm{W}$; alt. $\left.850-1350 \mathrm{~m}\right)$, situada no primeiro planalto paranaense, a cerca de 40-60 km ao sul da cidade de Curitiba, abrangendo parte dos municípios de São José dos Pinhais, Tijucas do Sul e Agudos do Sul (PARANÁ, 1987)(Figura 1).

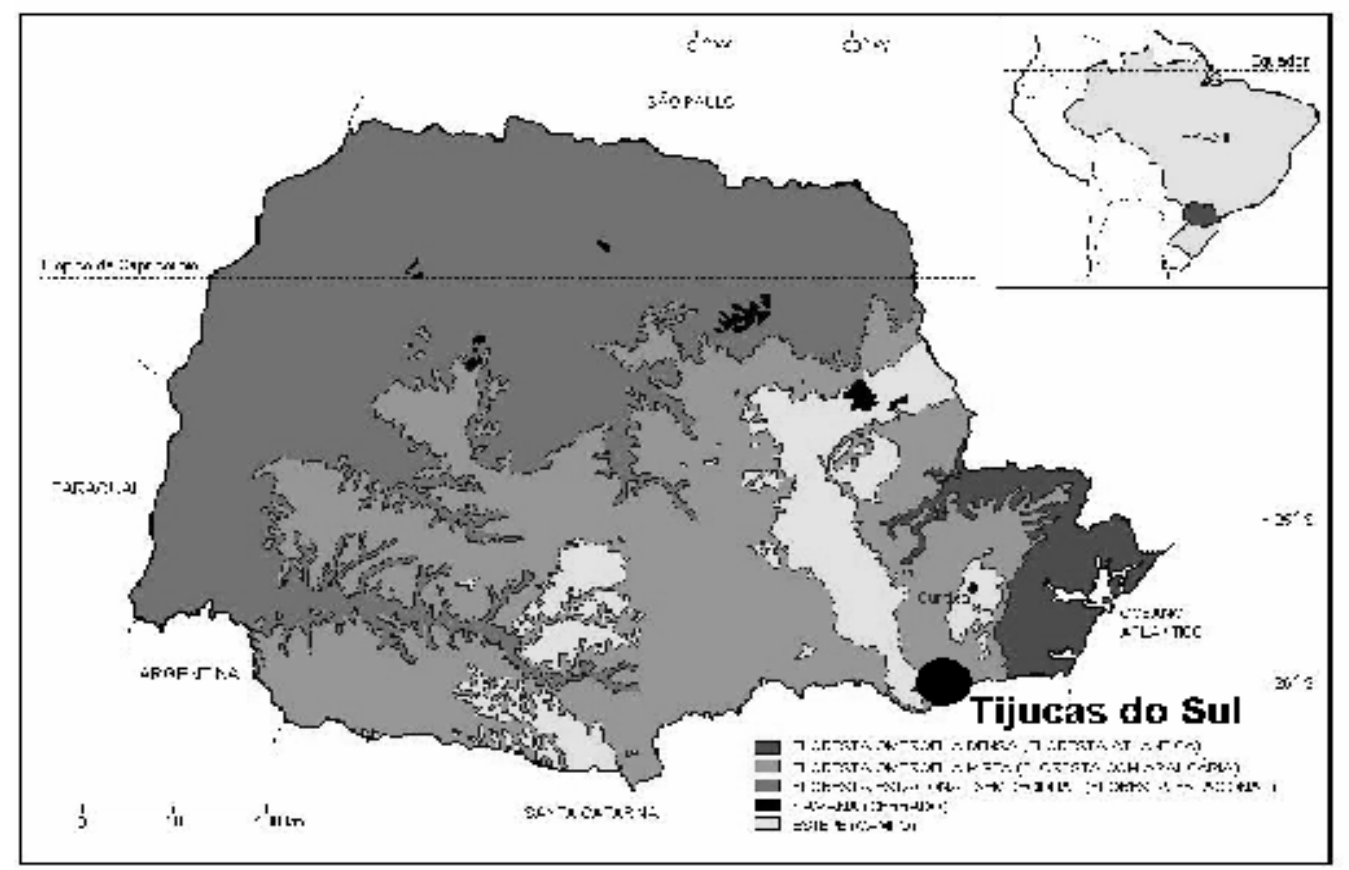

Fonte: MAACK, R. 1950, modificado por RODERJAN, C. V. et al (2002); inclusão de Tijucas do Sul pelos autores.

FIGURA 1: Localização do município de Tijucas do Sul, no estado do Paraná.

FIGURE 1: Location of Tijucas do Sul district in Paraná State.

A área foi adquirida originalmente pela Panagro Empreendimentos Florestais Ltda e foi cedida sob 
regime de comodato à Pontifícia Universidade Católica do Paraná. Nessa área está situado o Pró-Ação (Programa de Ação Comunitária e Ambiental), unidade de Tijucas do Sul.

O clima é do tipo subtropical úmido mesotérmico $(\mathrm{Cfb})$ com ocorrência de geadas severas e verões frescos (IAPAR, 1978). A média das temperaturas dos meses mais quentes é inferior a $22^{\circ} \mathrm{C}$ e a dos meses mais frios é inferior a $18^{\circ} \mathrm{C}$. Precipitações médias anuais são de $1.400 \mathrm{~mm}$ (Ferreira, 1999).

Dentro da propriedade em questão, foi localizada uma parcela de floresta, com cerca de 6 ha, para constituir-se de região amostral. Trata-se de um remanescente relictual, alterado pelo extrativismo, pastoreio de animais e atividades apícolas, porém uma parte mantém preservado o perfil característico de floresta de araucária, ou seja, estrato superior variando entre 20-25 metros e estrato emergente composto pelo pinheirodo-paraná (Liebsch, 2002).

\section{Estabelecimento das unidades amostrais}

Para estudo da vegetação e seu gradiente de conservação, foram alocadas duas parcelas de 100 X 100 m (1 ha cada). Na primeira parcela (área A), a vegetação primária foi muito alterada e a mata era utilizada como pastagem para o gado que utilizava as ervas do sub-bosque como alimento complementar à pastagem do campo limpo (Liebsch e Acra, 2002). Porém há 7 anos não apresenta mais interferência, restando apenas algumas árvores da mata original e um denso sub-bosque, dando aspecto de capoeirinha e capoeira, segundo classificação do IBGE (1992). A segunda parcela (área B), estava localizada no interior da floresta, onde a intervenção ficou restrita à retirada seletiva de madeira e a algumas trilhas de acesso à floresta e apresenta a estratificação evidente, mantendo características fisionômicas de Floresta Ombrófila Mista. As duas parcelas foram alocadas no mesmo fragmento distando $50 \mathrm{~m}$. uma da outra. A capoeira se localizava na parte mais externa do fragmento (área A), e a floresta (área B) no seu interior.

\section{Método de coleta}

As coletas se iniciaram em outubro de 2001 e se estenderam até setembro de 2002 . O material foi coletado somente em estado fértil e foi herborizado seguindo padrões tradicionais de herborização e exsicatagem. A identificação foi realizada no Herbário da Pontifícia Universidade Católica do Paraná (HUCP) e no Museu Botânico Municipal de Curitiba (MBM), com a utilização de chaves analíticas, comparação com as coleções do HUCP e MBM e consultas a especialistas. O material foi incorporado ao acervo do HUCP e MBM.

Somente foram coletados exemplares que pertenciam às seguintes categorias: indivíduos férteis com altura igual ou superior a $50 \mathrm{~cm}$ do solo e/ou indivíduos férteis com diâmetro à altura do peito (DAP) menor que $10 \mathrm{~cm}$. O levantamento florístico foi realizado por meio de coletas mensais, em que era percorrida toda a área, sendo utilizadas trilhas e estradas antigas, além de caminhos abertos no interior das formações.

\section{Parâmetros analisados}

Os dados foram avaliados quanto à riqueza para cada área estudada (área A e B), para o estudo da similaridade entre as áreas, foi utilizado o Índice de Similaridade Florística de Jaccard, esse índice é calculado de forma a correlacionar o número de espécies em comum entre as duas fisionomias florestas (capoeira e floresta), com o número total de espécies presentes em ambas.

\section{RESULTADOS}

Nos 12 meses de coletas nas duas áreas foram identificadas 89 espécies, pertencentes a 54 gêneros em 32 famílias botânicas, sendo 84 espécies na capoeira (62 exclusivas da capoeira) e 27 espécies na floresta (cinco exclusivas).

$\mathrm{Na}$ área da capoeira, as famílias, que apresentaram maiores riquezas, foram: Solanaceae (15 espécies), Asteraceae (14), Melastomataceae (13), Myrtaceae (sete), Lamiaceae e Poaceae com três espécies cada (Tabela 1). A família Solanaceae está representada com seis gêneros (Athenaea, Aureliana, Brunfelsia, Cestrum, Cyphomandra e Solanum). O gênero mais rico foi Solanum, com nove espécies (Tabela 2). A família Asteraceae presente com 14 espécies distribuídos em dez gêneros e ocorreu com exclusividade na capoeira. Melastomataceae foi a terceira mais rica, com três gêneros e 13 espécies, o gênero mais rico foi 
Leandra, com nove espécies. Essa família ocorreu em ambientes não muito abertos, em locais mais sombreados, assim como Myrtaceae (sete espécies). Duas famílias com três espécies se destacaram na capoeira, Lamiaceae e Poaceae, essas famílias foram encontradas em ambientes abertos e muito alterados, como na estrada e no apiário.

A área B (floresta) apresentou 27 espécies, sendo cinco espécies exclusivas da floresta, as espécies que só ocorrem na floresta pertencem às seguintes famílias: Euphorbiaceae, Melastomataceae, Myrtaceae, Rubiaceae e Tiliaceae. As famílias, que apresentaram maior riqueza na floresta, foram Melatomataceae e Solanaceae, com cinco espécies cada, Myrtaceae, com cinco espécies, seguidas por Euphorbiaceae, Flacourtiaceae, Moraceae, Meliaceae Piperaceae, Poaceae, Rubiaceae, Tiliaceae, Verbenaceae e Winteraceae, com uma espécie cada (Tabela 2). Foi observado que Melastomataceae parece bem adaptada a vida no interior da floresta, pois essa família, juntamente com Psychotria suterella (Rubiaceae), foi encontrada formando densos agrupamentos. Solanaceae está presente na floresta com três gêneros (Aureliana, Brunfelsia e Solanum), sendo que Solanum, com quatro espécies, foi o gênero mais rico. Foram encontradas cinco espécies de Myrtaceae, sendo Eugenia neoverrucosa exclusiva na floresta (Tabela 2).

A similaridade florística foi analisada utilizando o Índice de Similaridade Florística de Jaccard, o resultado obtido no presente estudo foi de $24,72 \%$.

TABELA 1: Famílias com maior riqueza, por área (área A: capoeira; área B: floresta). O número indica a quantidade de espécies.

TABLE 1: Family with larger richness, per area (area A: shrub; area B: forest). The value indicates the species number.

\begin{tabular}{l|c|c}
\hline Família & Área A & Área B \\
\hline Asteraceae & 14 & - \\
Commelinaceae & 2 & - \\
Euphorbiaceae & 1 & - \\
Lamiaceae & 3 & \\
Malvaceae & 2 & 6 \\
Melastomataceae & 13 & 5 \\
Myrtaceae & 7 & 1 \\
Piperaceae & 2 & 1 \\
Poaceae & 3 & - \\
Rosaceae & 2 & - \\
Rubiaceae & 2 & 6 \\
Solanaceae & 15 & 1 \\
Verbenaceae & 2 & \\
\hline
\end{tabular}

TABELA 2: Ocorrência das espécies na capoeira (área A) e floresta (área B) num fragmento de Floresta Ombrófila Mista em Tijucas do Sul.

TABLE 2: Occurrence of species in shrub (area A) and forest (area B) in fragment of Mixed Ombrophilous Forest in Tijucas do Sul district.

\begin{tabular}{|c|c|c|c|c|}
\hline Família & & Área A & Área B & Hábito* \\
\hline Asclepiadaceae & Asclepias curassavica L. & $\mathrm{X}$ & & $\mathrm{EV}$ \\
\hline \multirow[t]{7}{*}{ Asteraceae } & Austroeupatorium pictuatum (Malme) & $\mathrm{X}$ & & EV \\
\hline & R. M. King \& H. Robinson & & & \\
\hline & $\begin{array}{l}\text { Baccharis brachylaenoides DC. var. Polycephala } \\
\text { (Sch. Bip.) G. M. Barroso }\end{array}$ & $X$ & & $\mathrm{AB}$ \\
\hline & Baccharis trimera (Less) DC. & $\mathrm{X}$ & & $\mathrm{AB}$ \\
\hline & Baccharidastrum triplinerve (Less.) Cabrera & $\mathrm{X}$ & & $\mathrm{AB}$ \\
\hline & Chaptalia nutans (L.) Polak. & $\mathrm{X}$ & & EV \\
\hline & Eupatorium tweedianum Hook. et Arn. & $\mathrm{X}$ & & $\mathrm{AB}$ \\
\hline
\end{tabular}


TABELA 2: Continuação ...

TABLE 2: Continued ...

\begin{tabular}{|c|c|c|c|c|}
\hline Família & & Área A & Área B & Hábito* \\
\hline & Eupatorium $\mathrm{sp}$ & $\mathrm{X}$ & & $\mathrm{AB}$ \\
\hline & Jungia selowii Less & $\mathrm{X}$ & & $\mathrm{EV}$ \\
\hline & Mikania orleansensis Hieron. & $\mathrm{X}$ & & $\mathrm{EV}$ \\
\hline & Podocoma notobellidiastrum (Griseb.) Nesom & $X$ & & $\mathrm{EV}$ \\
\hline & Senecio icoglossus DC. & $X$ & & $\mathrm{EV}$ \\
\hline & Vernonia florida Gardner & $\mathrm{X}$ & & $\mathrm{AB}$ \\
\hline & Vernonia polyanthes Less & $X$ & & $\mathrm{AB}$ \\
\hline & Vernonia westiniana Less & $\mathrm{X}$ & & $\mathrm{AB}$ \\
\hline Anacardiaceae & Schinus terebinthifolius Raddi & $X$ & & $\mathrm{AV}$ \\
\hline Balsaminaceae & Impatiens walleriana Hook & $\mathrm{X}$ & & $\mathrm{EV}$ \\
\hline Berberidaceae & Berberis laurina Billb. & $X$ & & $\mathrm{AB}$ \\
\hline Bignoniaceae & Jacaranda puberula Cham. & $X$ & & AV \\
\hline Budlejaceae & Budleja brasiliensis Jacq. & $\mathrm{X}$ & & $\mathrm{AV}$ \\
\hline Caesalpiniaceae & Senna sp & $\mathrm{X}$ & & $\mathrm{AV}$ \\
\hline \multirow{2}{*}{ Commelinaceae } & Commelina erecta $\mathrm{L}$. & $X$ & & $\mathrm{EV}$ \\
\hline & Tradeschantia sp & $\mathrm{X}$ & & $\mathrm{EV}$ \\
\hline Cyperaceae & Fimbristylis autumnalis (L.) Roem \& Schult. & $X$ & & $\mathrm{EV}$ \\
\hline \multirow[t]{2}{*}{ Euphorbiaceae } & Croton ceanothifolius Baill. & $X$ & & $\mathrm{AB}$ \\
\hline & Croton celtidifolius Baill. & & $\mathrm{X}$ & AV \\
\hline Flacourtiaceae & Casearia sylvestris $\mathrm{Sw}$. & $X$ & $X$ & AV \\
\hline \multirow[t]{3}{*}{ Lamiaceae } & Hyptis lappulacea Mart ex Benth & $\mathrm{X}$ & & $\mathrm{EV}$ \\
\hline & Salvia melisseflora Benth. & $X$ & & $\mathrm{EV}$ \\
\hline & Ocimum selloi Benth. & $X$ & & $\mathrm{EV}$ \\
\hline Lithraceae & Heimia myrtifolia C. DC. & $X$ & & $\mathrm{EV}$ \\
\hline \multirow[t]{2}{*}{ Malvaceae } & Pavonia sepium A. St.-Hil. & $\mathrm{X}$ & & $\mathrm{EV}$ \\
\hline & Sida potentilloides St.-Hil. & $X$ & & $\mathrm{EV}$ \\
\hline Moraceae & Ficus luschnathiana (Miq.) Miq. & $\mathrm{X}$ & $\mathrm{X}$ & AV \\
\hline \multirow[t]{13}{*}{ Melastomataceae } & Leandra australis (Cham.) Cogn. & $X$ & $\mathrm{X}$ & $\mathrm{AB}$ \\
\hline & Leandra refracta Cogn. & $X$ & $\mathrm{X}$ & $\mathrm{AB}$ \\
\hline & Leandra regnellii (Triana) Cogn. & $X$ & $X$ & $\mathrm{AB}$ \\
\hline & Leandra sylvestris DC. & $X$ & $X$ & $\mathrm{AB}$ \\
\hline & Leandra xanthocoma (Naud.) Cogn. & $X$ & & $\mathrm{AB}$ \\
\hline & Leandra sp 1 & $X$ & & $\mathrm{AB}$ \\
\hline & Leandra sp 2 & $\mathrm{X}$ & & $\mathrm{AB}$ \\
\hline & Leandra $\mathrm{sp} 3$ & $X$ & & $\mathrm{AB}$ \\
\hline & Leandra sp 4 & $X$ & & $\mathrm{AB}$ \\
\hline & Miconia hyemalis Naud. & $X$ & & $\mathrm{AB}$ \\
\hline & Miconia petropolitana Cogn. & $X$ & $\mathrm{X}$ & $\mathrm{AV}$ \\
\hline & Miconia theaezans (Bonpl.) Cogn & $\mathrm{X}$ & $X$ & $\mathrm{AV}$ \\
\hline & Tibouchina cerastifolia (Naudin) Cogn. & $X$ & & $\mathrm{AB}$ \\
\hline Meliaceae & Cabralea canjerana (Vell.) Mart. & & $X$ & $\mathrm{AV}$ \\
\hline \multirow[t]{5}{*}{ Myrtaceae } & Eugenia neoverrucosa Sobral & & $X$ & $\mathrm{AV}$ \\
\hline & Gomidesia selowiana Berg & $\mathrm{X}$ & & AV \\
\hline & Myrceugenia cucullata Legrand & $X$ & $X$ & $\mathrm{AV}$ \\
\hline & Myrceugenia euosma (Berg) Legrand & $\mathrm{X}$ & & $\mathrm{AV}$ \\
\hline & Myrceugenia sp 1 & $X$ & $\mathrm{X}$ & $\mathrm{AV}$ \\
\hline
\end{tabular}


TABELA 2: Continuação ...

TABLE 2: Continued ...

\begin{tabular}{|c|c|c|c|c|}
\hline Família & & Área A & Área B & Hábito* \\
\hline & Myrceugenia sp 2 & $\mathrm{X}$ & $\mathrm{X}$ & $\mathrm{AV}$ \\
\hline & Myrceugenia sp 3 & $X$ & & $\mathrm{AV}$ \\
\hline & Myrtaceae 1 & $\mathrm{X}$ & $\mathrm{X}$ & AV \\
\hline Orchidaceae & Orchidaceae 1 & $\mathrm{X}$ & & EV \\
\hline \multirow[t]{2}{*}{ Piperaceae } & Piper gaudichaudianum Kunth & $\mathrm{X}$ & & $\mathrm{AB}$ \\
\hline & Piper xillosteoides (Kunth) Steud & $\mathrm{X}$ & $\mathrm{X}$ & $\mathrm{AB}$ \\
\hline \multirow[t]{3}{*}{ Poaceae } & Ichnanthus sp & $\mathrm{X}$ & & $\mathrm{EV}$ \\
\hline & Panicum maximum Jacq. & $\mathrm{X}$ & $\mathrm{X}$ & $\mathrm{EV}$ \\
\hline & Setaria sp & $X$ & & EV \\
\hline Polygalaceae & Polygala lancifolia A. St.-Hil. & $X$ & & EV \\
\hline \multirow[t]{2}{*}{ Rosaceae } & Rubus brasiliensis Mart. & $X$ & & $\mathrm{AB}$ \\
\hline & Rubus sellowii Koehne & $\mathrm{X}$ & & $\mathrm{AB}$ \\
\hline \multirow[t]{2}{*}{ Rubiaceae } & Psychotria stachyoides Benth. & $X$ & & $\mathrm{AB}$ \\
\hline & Psychotria suterella Müll. Arg. & & $\mathrm{X}$ & $\mathrm{AB}$ \\
\hline Sapindaceae & Allophyllus edulis (A. St.-Hil.) Radlk. & $\mathrm{X}$ & & $\mathrm{AV}$ \\
\hline \multirow{15}{*}{ Solanaceae } & Athenaea picta (Mart.) Sendtn. & $\mathrm{X}$ & & $\mathrm{AB}$ \\
\hline & $\begin{array}{l}\text { Aureliana fasciculata (Vell.) Sendtn var longifolia } \\
\text { (Sendtn.) Hunz \& Barbosa }\end{array}$ & $\mathrm{X}$ & $X$ & $\mathrm{AB}$ \\
\hline & Brunfelsia brasiliensis (Spreng) Smith \& Downs & $\mathrm{X}$ & $\mathrm{X}$ & $\mathrm{AB}$ \\
\hline & Cestrum amictum Schltdl. & $\mathrm{X}$ & & $\mathrm{AB}$ \\
\hline & Cyphomandra kleinii Smith \& Downs & $\mathrm{X}$ & & $\mathrm{AB}$ \\
\hline & Cyphomandra macrophylla Smith \& Downs & $\mathrm{X}$ & & $\mathrm{AB}$ \\
\hline & Solanum acerosum Sendtn. & $\mathrm{X}$ & $\mathrm{X}$ & $\mathrm{AB}$ \\
\hline & Solanum concinum Schott ex Sendtn. & $\mathrm{X}$ & & $\mathrm{AB}$ \\
\hline & Solanum diflorum Vell. & $\mathrm{X}$ & $\mathrm{X}$ & $\mathrm{AB}$ \\
\hline & Solanum johanae Bitter & $\mathrm{X}$ & $X$ & $\mathrm{AB}$ \\
\hline & Solanum lacerdae Dusén & $\mathrm{X}$ & $\mathrm{X}$ & $\mathrm{AB}$ \\
\hline & Solanum ranulatum Sendtn. & $X$ & & $\mathrm{AB}$ \\
\hline & Solanum reflexum Schrank & $\mathrm{X}$ & & $\mathrm{AB}$ \\
\hline & Solanum schwackeanum Smith \& Downs & $X$ & & $\mathrm{AB}$ \\
\hline & Solanum sp & $\mathrm{X}$ & & $\mathrm{AB}$ \\
\hline Tiliaceae & Triumpheta semitriloba Jacq. & & $\mathrm{X}$ & $\mathrm{EV}$ \\
\hline Thymelaeaceae & Daphnopsis racemosa Griseb. & $\mathrm{X}$ & & $\mathrm{AB}$ \\
\hline \multirow[t]{2}{*}{ Verbenaceae } & Lantana brasiliensis Link. & $X$ & $\mathrm{X}$ & $\mathrm{EV}$ \\
\hline & Lantana fucata Lindl. & $X$ & & $\mathrm{EV}$ \\
\hline Winteraceae & Drymis brasiliensis Miers & $X$ & $\mathrm{X}$ & $\mathrm{AV}$ \\
\hline Zingiberaceae & Hedychium coronarium Koen & $\mathrm{X}$ & & EV \\
\hline
\end{tabular}

Em que: * = Hábito $(\mathrm{AB}=$ arbusto; $\mathrm{AV}=$ árvore; $\mathrm{EV}=$ erva $)$.

\section{DISCUSSÃO}

A ação prolongada das atividades de criação de gado na área estudada refletiu na diminuição da cobertura do dossel. Tal situação propiciou a penetração de maior quantidade de luz até o solo, possibilitando o desenvolvimento de um estrato arbustivo com elevada cobertura e com a presença de espécies heliófilas (Brokaw apud Armelin e Mantovani, 2001).

Segundo Tabarelli e Mantovani (1999), na Floresta Atlântica, os gêneros Miconia, Leandra, Tibouchina e Solanum são arbustos pioneiros de ciclo de vida curto. Tal característica pode favorecer a alta riqueza desses gêneros, pois no remanescente em estudo, estes estão entre os mais freqüentes, com três 
espécies de Miconia, nove Leandra, nove de Solanum e uma espécie de Tibouchina. Teixeira e Mantovani (1998), em um fragmento florestal na Região Metropolitana de São Paulo, encontraram na borda de um fragmento uma ampla distribuição das famílias Asteraceae, Poaceae e Solanaceae, corroborando com esse estudo, onde essas famílias são típicas de ambientes alterados e bordas de fragmentos.

O número de espécies na capoeira foi maior que na área com floresta. Essa relação está de acordo com Oliveira e Rotta (1982) que, comparando uma floresta intensamente explorada, com uma área conservada em Colombo-PR, encontraram 145 espécies, sendo 103 pertencentes à área mais explorada. A presença de distúrbios humanos, como a retirada indiscriminada de madeira, pode favorecer a manutenção momentânea de um elevado número de espécies, sobretudo as intolerantes à sombra, que necessitam de grandes aberturas no dossel para o seu desenvolvimento (Vieira e Hosokawa, 1989). Entretanto, a retirada de madeira, como forma de manutenção de uma grande diversidade de espécies, deve ser vista com extremo cuidado (Werneck et al, 2000), pois esses distúrbios alteram a estrutura e a composição florística original e, portanto, a dominância local de espécies, ocasionando danos à biodiversidade (Vidal et al, 1998).

Os indivíduos de Cyphomandra kleinii, Jungia sellowii e Solanum acerosum morreram após a floração e frutificação no final do verão, vindo a aparecer no mês de setembro e outubro (primavera), florescendo em novembro. Isso demonstra que essas espécies são de ciclo anual, típico de espécies de capoeira. (Werneck et al, 2000).

Segundo Cestaro et al. (1986), Chaptalia nutans e Pavonia sepium são espécies características de ambientes alterados, esse fato é observado na área de estudo, uma vez que essas espécies foram encontradas nas áreas mais degradas e nas margens de estradas.

$\mathrm{Na}$ área de estudo, foi constatada a presença de duas espécies exóticas: Impatiens walleriana e Hedychium coronarium. A ocorrência dessas espécies aponta um aspecto negativo, pois compromete a disseminação das espécies características do local e sua sobrevivência (Cervi et al., 1989). Impatiens walleriana (beijinho) foi encontrado em estado fértil o ano todo.

A sucessão secundária é o processo que ocorre em áreas previamente ocupadas por uma comunidade vegetal, após um impacto natural ou antrópico (Amador e Viana, 2000). Impacto esse que pode ser efetuado pela exploração seletiva de madeiras e pelo corte raso para a realização da agricultura itinerante, o que causa a abertura de grandes clareiras e o surgimento da vegetação secundária (Rondon-Neto et al., 2000). Esse processo parece estar ocorrendo na área mais preservada do fragmento, pois segundo Klein (1960); Britez et al. (1995) e Dislich et al. (2001): Drymis brasiliensis, Casearia sylvestris, Schinus terebinthifolius e Myrceugenia euosma, pertencem ao grupo das principais espécies arbóreas na sucessão secundária.

As famílias mais ricas na floresta foram Melastomataceae, Solanaceae e Myrtaceae que também são freqüentes em povoamentos de florestas plantadas (Nappo et al., 2000). Esses autores estudaram a estrutura do sub-bosque em povoamentos de bracatinga (Mimosa scabrela), encontrando a família Asteraceae com maior riqueza específica ( 8 espécies), seguida por Melastomataceae (6 espécies), Myrtaceae e Solanaceae (5 espécies cada).

Para Nascimento et al (2001), a Floresta Ombrófila Mista constitui um importante centro de dispersão da família Myrtaceae, sendo observado desde árvores de grande porte até arvoretas e arbustos que habitam o sub-bosque. Neste estudo, a diversidade de espécies dessa família é bastante expressiva (cinco espécies distribuídas por toda floresta) o que corrobora com trabalhos anteriores de Rambo (1951); Klein (1984); Jarenkow (1985) e Nascimento et al. (2001).

A abertura de clareiras é o principal fator para que diversas espécies existam na floresta, sendo renovadas e sustentadas pela dinâmica de perda de indivíduos mais velhos, e a ocupação de espécies pioneiras, colonizadoras de grandes clareiras (Pizatto, 1999; Brokaw apud Armelin e Mantovani, 2001; Werneck et al, 2000). Foi observado, na área B, que diversas espécies típicas de borda (Solanum acerosum,Solanum diflorum, Solanum lacerdae e Solanum reflexum) apareceram nas trilhas abertas no interior da floresta.

Psychotria suterella caracterizou-se por estar amplamente distribuída no interior da floresta mais densa. Essa espécie, segundo Armelin e Mantovani (2001); Lacerda et al. (1999), essa espécie é comum no 
sub-bosque da Floresta Ombrófila Densa e é caracterizada como ciófita. O presente estudo corrobora com essa característica e confirma essa espécie para o sub-bosque na Floresta Ombrófila Mista.

Britez et al. (1995) constataram que, em um remanescente florestal no município de São Mateus do Sul-PR, o sub-bosque é constituído sobretudo por erva-mate (Ilex paraguariensis), no presente estudo não se observa essa espécie. Apesar da capoeira e floresta constituírem características de sucessão secundária, não se justifica a ausência dessa espécie na área.

O Índice de Similaridade de Jaccard obtido no presente estudo foi baixo em se tratando de duas áreas muito próximas. Segundo Mueller-Dombois e Ellemberg (1974), para duas formações florestais serem consideradas similares, o Índice de Similaridade de Jaccard deve ser superior a 25\%. De acordo com Oliveira e Rotta (1982), fatores que promovem resultados de índices muito baixos entre algumas parcelas de amostragens poder ser: diferenças de solo, exploração concentrada em parte da área ou até diferença de sítio. O segundo fator parece ser o que mais contribuiu para esse valor baixo de similaridade florística.

\section{CONSIDERAÇÕES FINAIS}

De acordo com este estudo, o remanescente apresenta sinais de regeneração e recuperação das suas características danificadas pelas atividades humanas, isto é, a diminuição no interior do fragmento das espécies pioneiras. Para que isso ocorra com sucesso é necessário traçar metas e estratégias de conservação e proteção desse fragmento florestal. A preservação de fragmentos vizinhos ao Capão do Pró-Ação, mesmo que em diversos níveis de perturbações, pode ser também extremante importante, pois contribuiria para a manutenção da sua diversidade de espécies e para recuperação da flora da região.

\section{AGRADECIMENTOS}

Os autores agradecem ao Professor, Dr., Sylvio Pellico Neto, pela viabilidade do auxílio financeiro e correção do abstract. A Arthur Ângelo Bispo de Oliveira pela correção do original. A Carlyle Santim Sguassabia, Daniel Carvalho Carneiro e Daniel Isolani, pelo auxílio nas coletas. Ao Engenheiro Florestal, MSc., Alexandre Koehler e à Bióloga, MSc., Marília Borgo. Ao Dr., Gerdt G. Hatschbach, ao Botânico Osmar dos Santos Ribas e ao Professor, Dr., Renato Goldenberg, pela identificação de alguns exemplares botânicos.

\section{REFERENCIAS BIBLIOGRAFICAS}

AMADOR, D. B.; VIANA, V. M. Dinâmica de "capoeiras baixas" na restauração de um fragmento florestal. Scientia Forestalis, Piracicaba, n. 57, p. 69-85, 2000.

ARMELIN, R. S.; MANTOVANI, W. Definições de clareira natural e suas implicações no estudo da dinâmica sucessional em florestas. Rodriguésia, Rio de Janeiro, v. 52, n. 81, p. 5-15, 2001.

BACKES, A. Condicionamento climático e distribuição geográfica de Araucaria angustifolia (Bert.) O. Kuntze no Brasil. Pesquisas-Botânica, São Leopoldo, n. 39, p. 5-39, 1988.

BACKES, A. Condicionamento climático e distribuição geográfica de Araucaria angustifolia no Brasil - II. Pesquisas-Botânica, São Leopoldo, n. 49, p. 31-51, 1999.

BACKES, A.; FERNANDES, A. V.; ZENI, D. J. Produção de folhedo em uma floresta com Araucaria angustifolia no sul do Brasil. Pesquisa-Botânica, São Leopoldo, n. 50, p. 97-117, 2000.

BRITEZ, R. M.; CASTELLA, P. R.; TIEPOLO, G. PIRES, L. A. Estratégias de conservação da Floresta de Araucária para o Estado do Paraná - Diagnóstico da vegetação. In: CONGRESSO BRASILEIRO DE UNIDADES DE CONSERVAÇÃO, 2., 2000, Campo Grande - MS. Anais... Campo Grande - MS, 2000. p. 731-737.

BRITEZ, R. M.; SILVA, S. M.; SOUZA, W. S.; MOTTA, J. T. W. Levantamento florístico em Floresta Ombrófila Mista. São Mateus do Sul, Paraná, Brasil. Arq. Biol. Tecnol., Curitiba, v. 38, n. 4, p. 1147-1161, 1995.

CERVI, A. C.; PACIORNIK, E. F.; VIEIRA, R. F.; MARQUES, L. C. Espécies vegetais de um remanescente de floresta de Araucária (Curitiba, Brasil): Estudo preliminar I. Acta Biol. Par., Curitiba, v. 18, n. 1/4, p. 73-114, 1989.

CESTARO, L. A.; WAECHTER, J. L.; BAPTISTA, L. R. M. Fitossociologia do estrato herbáceo da mata de araucária da Estação Ecológica de Aracuri, Esmeralda, RS. Hoehnea, São Paulo, v. 13, p. 59-72, 1986. 
DISLICH, R.; CERSÓSIMO, L.; MANTOVANI, W. Análise da estrutura de fragmentos florestais no Planalto Paulistano - SP. Revta. Brasil. Bot., São Paulo, v. 24, n. 3, p. 321-332, 2001.

FERNANDES, A. V.; BACKES, A. Produtividade primária em floresta com Araucaria angustifolia no Rio Grande do Sul. Iheringia - Botânica, Porto Alegre, v. 51, n. 1, p. 63-78, 1998.

FERREIRA, J. C. V. O Paraná e seus municípios. 2. ed. Cuiabá: Memória do Brasil, 1999. p. 483-484.

GERHARDT. E. J.; FINGER, C. A. G.; LONGHI, S. J.; SCHUMACHER, M. V. Contribuição da análise multivariada na classificação de sítios em povoamentos de Araucaria angustifólia (Bert.) O. Ktze., baseada nos fatores físicos e morfológicos do solo e no conteúdo de nutrientes da serrapilheira. Ciência Florestal, Santa Maria, v. 11, n. 2, p. 4157, 2001.

IAPAR, Cartas climáticas básicas do Estado do Paraná. Londrina: Fundação Instituto Agronômico do Paraná, 1978. $38 \mathrm{p}$.

IBGE. Manual técnico da vegetação brasileira. Rio de Janeiro: IBGE, 1992. 92p. (Serie Manuais Técnicos em Biociências, v. 1).

JARENKOW, J. A. Composição Florística e estrutura da mata com Araucária na estação ecológica de Aracuri, Esmeralda, Rio Grande do Sul. 1985. 82 p. Dissertação (Mestrado em Botânica) - Universidade Federal do Rio Grande do Sul, Porto Alegre, 1985.

JARENKOW, J. A.; BAPTISTA, L. R. M. Composição florística e estrutura da Mata com Araucária na Estação Ecológica de Aracuri, Esmeralda, RS. Napaea, Porto Alegre, n. 3, p. 9-18, 1987.

KLEIN, R. M. O aspecto dinâmico do pinheiro brasileiro. Sellowia, Itajaí, v. 12, n. 12, p. 17-44. 1960.

KLEIN, R. M. Aspectos dinâmicos da vegetação do sul do Brasil. Sellowia, Itajaí, v. 36, n. 36, p. 5-54, 1984.

KOEHLER, A.; PÉlliCO NETO, S.; SANQUETA, C. R. Análise da estrutura de uma Floresta Ombrófila Mista semidevastada, Fazenda Gralha Azul, Região Metropolitana de Curitiba, com implicações ao manejo. Revista Acadêmica, Curitiba, v. 9, n. 1, p. 37-60, 1998.

LACERDA, A. E. B.; KUNIYOSHI, Y.; GALVÃO, F. Evidências de contato entre a Floresta Ombrófila e Mista Mananciais da Serra - Piraquara - PR. In: CONGRESSO NACIONAL DE BOTÂNICA, 50., 1999, Blumenau. Anais... Blumenau: SBB, 1999. p. 272.

LIEBSCH, D. Levantamento da vegetação de sub-bosque de um remanescente de Floresta Ombrófila Mista em Tijucas do Sul, Paraná, Brasil. 2002. 40 p. Monografia (Bacharelado em Biologia) - Pontifícia Universidade Católica do Paraná, Curitiba, 2002

LIEBSCH. D.; ACRA. L. A. Asteraceae Dumortier (=Compositae Giseke) de um Capão de Floresta Ombrófila Mista, da Área do PRÓAÇÃO/PUCPR, Município de Tijucas do Sul, Paraná, Brasil. In: BIOCEC, 3., 2002, Curitiba. Anais...Curitiba: Champagnat, 2002, p.25.

LINDMANN, C. A. A vegetação no Rio Grande do Sul. Belo Horizonte: EDUSP, 1974. 356 p.

LONGHI, S. J. A estrutura de uma floresta natural de Araucaria angustifolia (Bert.) O. Ktze, no sul do Brasil. 1980. 198 p. Dissertação (Mestrado em Ciência Florestal) - Universidade Federal do Paraná, Curitiba, 1980.

MAACK. R. Geografia Física do Estado do Paraná. Curitiba: José Olympio, 1968. 442 p.

MUELER-DOMBOIS, D.; ELLEMBERG, H. Aims and methods of vegetation ecology. New York: J. Wiley \& Sons, 1974. 574p.

NAPPO, M. E.; OLIVEIRA-FILHO, A. T.; MARTINS, S. V. A estrutura do sub-bosque de povoamentos homogêneos de Mimosa scabrella Bentham, em área minerada, em Poços de Caldas, MG. Ciência Florestal, Santa Maria, v. 10, n. 2, p. 17-29, 2000.

NASCIMENTO, A. R. T.; LONGHI, S. J.; BRENA, D. A. Estrutura e padrões de distribuição espacial de espécies arbóreas em uma amostra de floresta Ombrófila Mista em Nova Prata, RS. Ciência Florestal, Santa Maria, v. 11, n. 1, p. 105-119, 2001.

NEGRELlE, R. A. B.; SILVA, F. C. Fitossociologia de um trecho de floresta com Araucaria angustifolia (Bert.) O. Ktze. no município de Caçador-SC. Boletim de Pesquisas Florestais, Colombo, n. 24/25, p. 37-54, 1992.

OLIVEIRA, R. J.; MANTOVANI, W.; MELO, M. M. R. F. Estrutura do componente arbustivo-arbóreo da floresta Atlântica de encosta, Peruíbe, SP. Acta Bot. Bras., Rio de Janeiro, v. 15, n. 3, p. 391-412, 2001. 
OLIVEIRA, Y. M. M.; ROTTA, E. Levantamento da estrutura horizontal de uma mata de Araucária do primeiro planalto paranaense. Bol. Pesq. Flor., Curitiba, v. 4, p. 1-46, 1982.

PARANÁ. Atlas do Estado do Paraná. Curitiba: Instituto de Terras, Cartografias e Florestas, 1987. 73 p.

PIZATTO, W. Avaliação biométrica da estrutura e da dinâmica de uma Floresta Ombrófila Mista em São João do Triunfo-PR: 1995 a 1998. 1999. 170 p. Dissertação (Mestrado em Ciência Florestal) - Universidade Federal do Paraná, Curitiba, 1999.

RAMBO, B. O elemento andino no pinhal rio-grandense. Anais Botânicos do Herbário Barbosa Rodrigues, Itajaí, v. 3, n.3, p. 3-39, 1951.

REITZ, R.; KLEIN, R. M. Araucariáceas. Itajaí: Herbário Barbosa Rodrigues, 1966. 63 p. (Flora Ilustrada Catarinense).

RODERJAN, C. V.; GALVÃO, F.; KUNIYOSHI, Y. S.; HATSCHBACH, G. G. As unidades fitogeográficas do estado do Paraná, Brasil. Ciência \& Ambiente, Santa Maria, n. 24, p. 78-118, 2002.

RONDON-NETO, R. M.; BOTELHO, S. A.; FONTES, M. A. L.; DAVIDE, A. C.; FARIA, J. M. R. Estrutura e composição florística da comunidade arbustivo-arbórea de uma clareira de origem antrópica, em uma Floresta Estacional Semidecídua Montana, Lavras-MG, Brasil. Cerne, Lavras, v. 6, n. 2, p. 79-94, 2000.

ROSEIRA, D. S. Composição florística fitossociológica do bosque com Araucaria angustifolia (Bert.) O. Kuntze no parque Estadual João Paulo II, Curitiba, Paraná. 1990. 107 p. Dissertação (Mestrado em Botânica) Universidade Federal do Paraná, Curitiba, 1990.

TABARELLI, M.; MANTOVANI, W. Clareiras naturais e a riqueza de espécies pioneiras em uma Floresta Atlântica Montana. Ver. Brasil. Bio., São Paulo, v.59, n.2, p.251-261, 1999.

TEIXEIRA, C. V.; MANTOVANI, W. Vegetação na borda de um fragmento florestal na área metropolitana de São Paulo, SP. Série Técnica IPEF, Piracicaba, v. 12, n. 32, p.133-148, 1998.

VIDAL, E.; VIANA, V. M.; BAPTISTA, J. L. F. Efeitos da exploração madeireira predatória e planejada sobre a diversidade de espécies da Amazônia oriental. Revista Árvore, Viçosa, v. 22, p. 503-520, 1998.

VIEIRA, G.; HOSOKAWA, R. T. Composição florística da vegetação da regeneração natural, 1 ano após diferentes níveis de exploração de uma floresta tropical úmida. Acta Amazônica, Manaus, v. 19, p. 401-413, 1989.

WERNECK, M. S.; FRANCESCHINELLI, E. V.; TAMEIRÃO-NETO, E. Mudanças na florística e estrutura de uma floresta decídua durante um período de quatro anos (1994-1998), na região do Triângulo Mineiro, MG. Rev. Brasil. Bot., São Paulo, v. 23, n. 4, p. 401-413, 2000. 